\title{
Decoding Collagen Triple Helix Stability by Means of Hybrid DFT Simulations
}

\author{
Michele Cutini, ${ }^{1 *}$ Massimo Bocus ${ }^{1,2}$, and Piero Ugliengo ${ }^{1 *}$ \\ ${ }^{1}$ University of Torino, Department of Chemistry and NIS (Nanostructured Interfaces \\ and Surfaces) Center, Via P. Giuria 7, 10125 Turin-ITALY \\ ${ }^{2}$ Present address: University of Ghent, Center for Molecular Modeling, \\ Technologiepark 46, 9052 Zwijnaarde, Belgium \\ *e-mail:michele.cutini@unito.it; piero.ugliengo@unito.it
}

Supporting Information 
CRYSTAL17 Input file for the DU conformation of the GPP Collagen

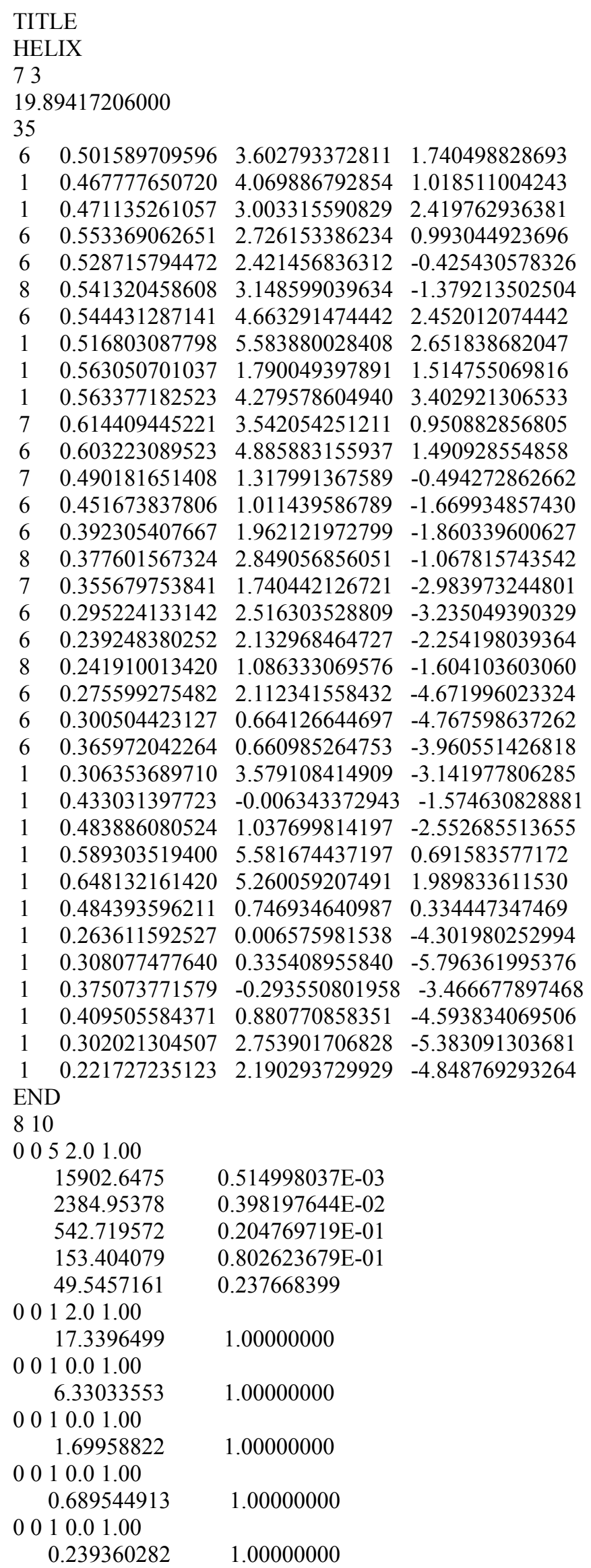




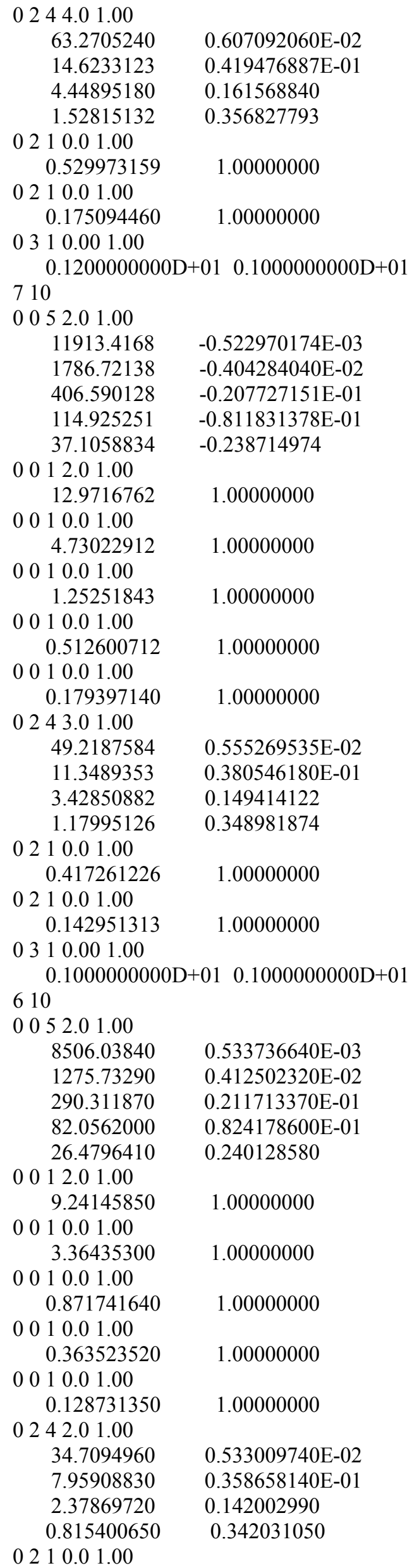




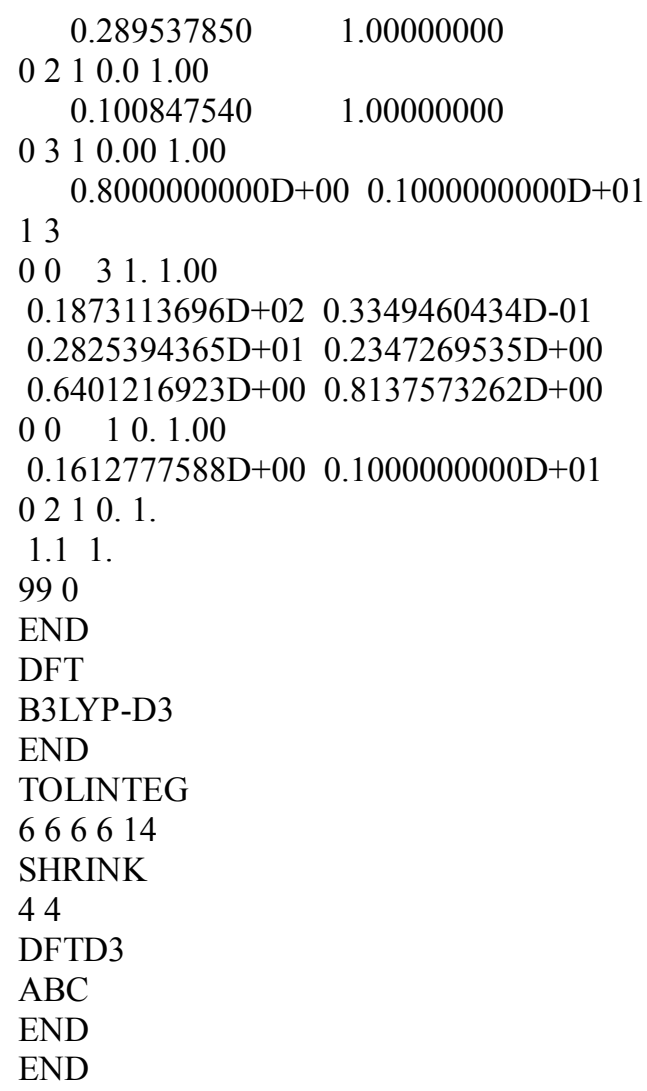


Table S1. Geometrical parameters and energies of the GPP triple helix (COL) models. The name indicates the Pro (X)/Pro (Y) puckering conformation, respectively. The angles are expressed in degrees, the hydrogen bond $(\mathrm{Hb})$ and cell lengths in Ångstrom, whilst the energies in $\mathrm{kJ} \cdot \mathrm{mol}^{-1} \cdot$ triplet $^{-}$ 1.

\begin{tabular}{|c|c|c|c|c|c|c|c|c|c|c|c|c|c|c|}
\hline & & "Gly & & & & $\overline{(X)}$ & & & & (Y) & & & & \\
\hline name & $\varphi$ & $\psi$ & $\omega$ & $\varphi$ & $\psi$ & $\omega$ & $\chi^{2}$ & $\varphi$ & $\psi$ & $\omega$ & $\chi^{2}$ & $\mathrm{Hb}$ & cell & $\Delta \mathrm{E}$ \\
\hline$D D$ & -70.4 & 174.6 & 176.2 & -69.0 & 163.6 & 168.6 & -35.9 & -57.7 & 155.2 & 170.8 & -37.2 & 2.018 & 19.973 & 1.57 \\
\hline$D U$ & -70.6 & 177.9 & 175.8 & -69.9 & 166.2 & 165.4 & -36.5 & -56.1 & 156.7 & 167.6 & 33.2 & 2.074 & 19.894 & 0.00 \\
\hline$U D$ & -76.3 & 177.7 & 174.8 & -61.5 & 158.5 & 165.3 & 33.1 & -61.7 & 157.9 & 179.2 & -37.3 & 1.976 & 19.955 & 6.42 \\
\hline$U U$ & -74.0 & 180.1 & 175.3 & -65.4 & 164.4 & 161.8 & 30.8 & -56.7 & 157.3 & 170.3 & 32.9 & 2.069 & 19.868 & 6.88 \\
\hline \multirow[t]{2}{*}{$E X P^{47}$} & -71.7 & 175.9 & 179.7 & -74.5 & 164.3 & 176.0 & All-D & -60.1 & 152.4 & 175.4 & All-U & & & \\
\hline & \pm 3.7 & \pm 3.1 & \pm 2.0 & \pm 2.9 & \pm 4.1 & \pm 2.5 & & \pm 3.6 & \pm 2.6 & \pm 3.4 & & & & \\
\hline \multirow[t]{2}{*}{$E X P^{48}$} & -74.6 & 176.7 & 177.1 & -76.6 & 163.2 & 178.9 & All-D & -60.8 & 153.4 & 176.9 & $6 \mathrm{U} / 1 \mathrm{D}$ & 2.17 & 20.25 & \\
\hline & & & & & & & & & & & & \pm 0.05 & & \\
\hline
\end{tabular}

Table S2. Geometrical parameters and energies of the GPO triple helix models. The name indicates the Prox $/$ Proy puckering conformation, respectively, and the $\mathrm{OH}$ group orientation. $\mathrm{Hb}$ is the interstrand hydrogen bond between Gly and Prox. The angles are expressed in degrees, the hydrogen bond (Hb) and cell lengths in Ångstrom whilst the energies in $\mathrm{kJ} \cdot \mathrm{mol}^{-1} \cdot$ triplet $^{-1}$.

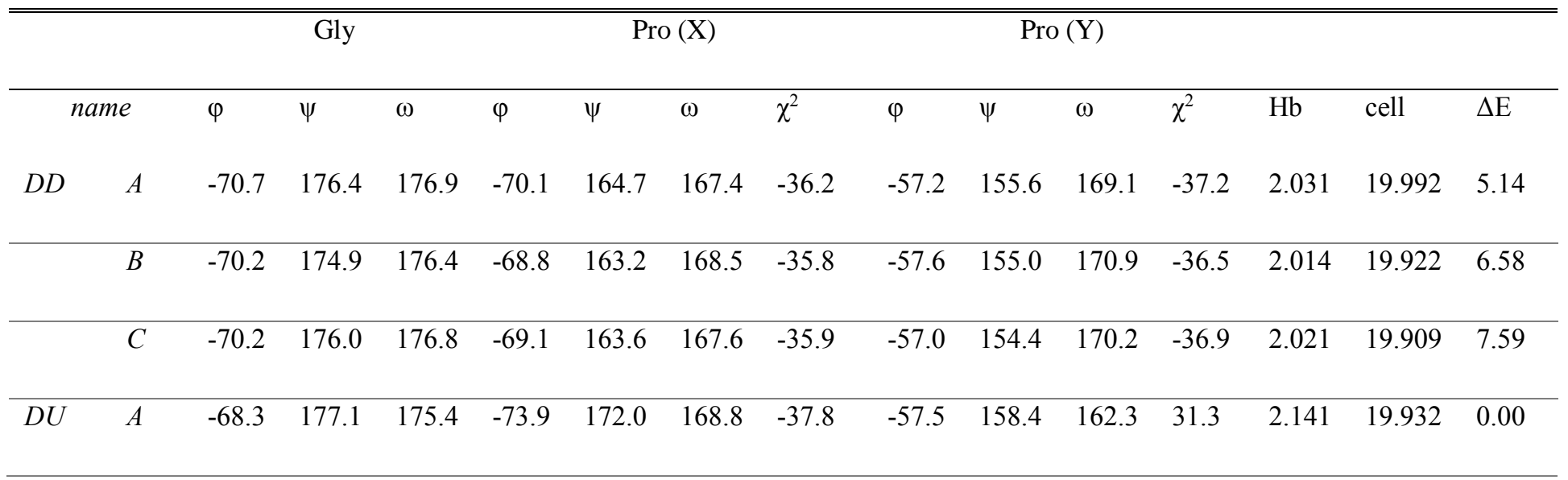




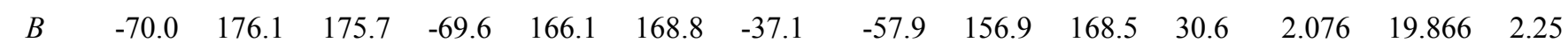

\begin{tabular}{|c|c|c|c|c|c|c|c|c|c|c|c|c|c|c|c|}
\hline & $C$ & -69.8 & 176.6 & 176.1 & -71.8 & 167.6 & 169.8 & -36.2 & -59.0 & 156.8 & 167.9 & 30.0 & 2.063 & 19.984 & 5.55 \\
\hline \multirow[t]{3}{*}{$U D$} & $A$ & -76.6 & 179.0 & 175.6 & -63.0 & 160.2 & 164.9 & 32.4 & -61.9 & 158.6 & 177.6 & -37.0 & 1.990 & 20.011 & 10.00 \\
\hline & $B$ & -75.3 & 178.4 & 175.0 & -62.1 & 159.6 & 164.9 & 32.8 & -61.0 & 157.8 & 177.4 & -36.8 & 1.995 & 19.878 & 11.65 \\
\hline & $C$ & -75.9 & 179.1 & 175.6 & -62.2 & 159.2 & 164.4 & 32.7 & -61.0 & 157.0 & 178.0 & -36.9 & 1.985 & 19.907 & 12.17 \\
\hline \multirow[t]{3}{*}{$U U$} & $A$ & -71.2 & 179.9 & 174.1 & -67.7 & 170.3 & 161.3 & 29.2 & -55.1 & 158.6 & 162.9 & 33.9 & 2.176 & 19.803 & 1.92 \\
\hline & $B$ & -73.3 & 178.6 & 173.2 & -61.5 & 161.8 & 162.6 & 31.4 & -57.4 & 157.6 & 172.1 & 31.6 & 2.063 & 19.711 & 5.67 \\
\hline & $C$ & -74.2 & 179.5 & 174.7 & -63.5 & 161.9 & 162.8 & 32.5 & -58.0 & 156.9 & 173.2 & 31.4 & 2.021 & 19.850 & 6.34 \\
\hline
\end{tabular}

\begin{tabular}{lllllllllllll}
\hline EXP $^{52}$ & -71.1 & 173.4 & 178.7 & -70.0 & 162.3 & 172.3 & $4 \mathrm{D} / 3 \mathrm{U}$ & -57.0 & 149.6 & 174.6 & All U & 19.89 \\
& & & & & & & & & & & \\
& \pm 1.8 & \pm 1.3 & \pm 1.2 & \pm 1.5 & \pm 1.2 & \pm 1.1 & & \pm 1.4 & \pm 1.3 & \pm 1.3 & &
\end{tabular}




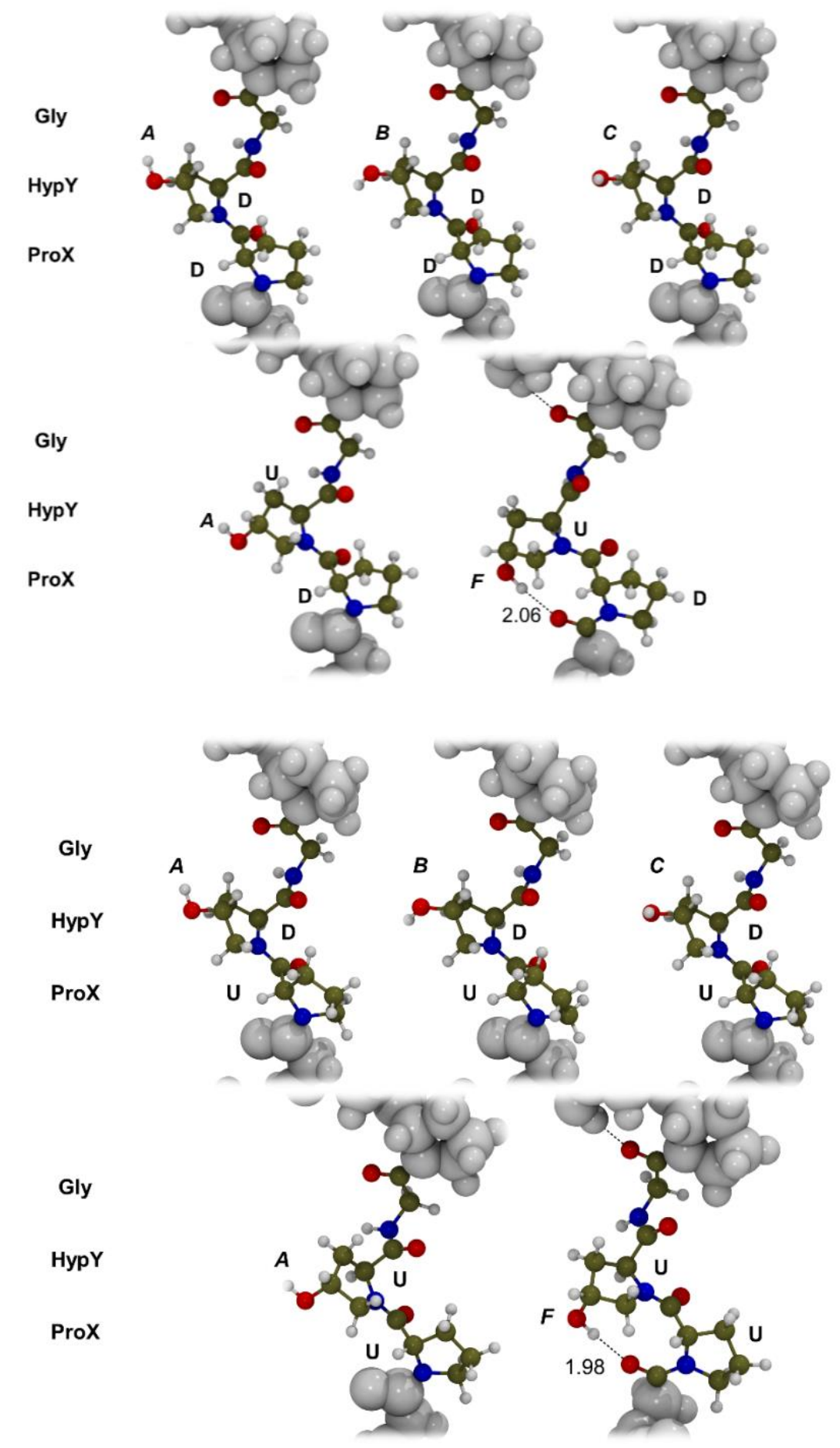

Figure S1. Equilibrium geometries of the GPO single strand in the PPII conformation. The amino acid triplet is highlighted in ball and stick representation. The letters close to the hydroxyl group indicates its orientation and those close to the proline rings the respective puckering. The hydrogen bond length, in $\AA$, is reported for the models in which is particular strong (DU_F and UU_F). 
Table S3. Geometrical parameters and energies of the GPO single strands models. The abbreviations stay for Prox/Hypy up (U) and down (D) respectively. $\mathrm{Hb}$ is the possible additional intramolecular hydrogen bond between the hydroxyproline $\mathrm{OH}$ and the nearest carbonyl. The angles are expressed in degrees, the hydrogen bond between the $\mathrm{OH}$ and the nearest carbonyl $(\mathrm{Hb})$ and cell lengths in Ångstrom whilst the energies in $\mathrm{kJ} \cdot \mathrm{mol}^{-1} \cdot$ triplet $^{-1}$.

\begin{tabular}{|c|c|c|c|c|c|c|c|c|c|c|c|c|c|c|c|}
\hline \multicolumn{6}{|c|}{ Gly } & \multicolumn{3}{|c|}{ Pro $(\mathrm{X})$} & \multicolumn{4}{|c|}{ Нyp (Y) } & \multirow[b]{2}{*}{$\mathrm{Hb}$} & \multirow[b]{2}{*}{ cell } & \multirow[b]{2}{*}{$\Delta \mathrm{E}$} \\
\hline$n a$ & & $\varphi$ & $\psi$ & $\omega$ & $\varphi$ & $\psi$ & $\omega$ & $\chi^{2}$ & $\varphi$ & $\psi$ & $\omega$ & $\chi^{2}$ & & & \\
\hline \multirow[t]{3}{*}{$D D$} & $A$ & -81.1 & 163.7 & 172.1 & -79.3 & 161.4 & 162.6 & -35.1 & -68.3 & 161.6 & 158.8 & -35.9 & 4.793 & 9.287 & 7.02 \\
\hline & $B$ & -81.9 & 161.8 & 174.2 & -78.6 & 160.4 & 163.1 & -35.7 & -67.6 & 159.6 & 159.8 & -35.2 & - & 9.286 & 9.10 \\
\hline & $C$ & -81.3 & 162.8 & 173.9 & -79.4 & 159.9 & 163.1 & -35.4 & -67.8 & 159.1 & 160.4 & -35.3 & - & 9.297 & 10.35 \\
\hline \multirow[t]{2}{*}{$D U$} & $A$ & -80.4 & 162.5 & 175.5 & -81.0 & 157.7 & 161.0 & -35.2 & -59.6 & 154.7 & 157.3 & 34.0 & & & 12.9 \\
\hline & $F$ & -65.8 & 141.2 & 178.0 & -68.7 & 121.9 & -173.6 & -35.3 & -68.4 & 148.0 & -169.7 & 41.8 & 2.056 & 8.754 & 4.44 \\
\hline \multirow[t]{3}{*}{$U D$} & $A$ & -79.5 & 160.6 & 169.1 & -75.3 & 162.3 & 162.3 & 21.1 & -68.4 & 162.9 & 158.6 & -36.0 & 4.726 & 9.203 & 14.31 \\
\hline & $B$ & -78.8 & 156.6 & 171.0 & -73.6 & 160.8 & 163.9 & 24.0 & -67.3 & 160.0 & 159.7 & -35.5 & - & 9.171 & 16.35 \\
\hline & $C$ & -79.0 & 159.1 & 170.8 & -75.3 & 160.9 & 163.5 & 21.8 & -67.9 & 160.2 & 160.1 & -35.5 & - & 9.204 & 17.78 \\
\hline \multirow[t]{2}{*}{$U U$} & $A$ & -73.3 & 149.1 & 172.7 & -70.8 & 151.9 & 164.2 & 30.7 & -57.5 & 150.6 & 161.2 & 35.9 & - & 9.010 & 20.07 \\
\hline & $F$ & -60.6 & 125.8 & 170.6 & -57.3 & 122.1 & -172.8 & 38.7 & -70.2 & 152.9 & -175.5 & 41.5 & 1.979 & 8.681 & 0.00 \\
\hline
\end{tabular}

